\title{
Iron Deficiency and Renal Development in the Newborn Rat
}

\author{
KERI A. DRAKE, MOLLY J. SAUERBRY, SHARON E. BLOHOWIAK, KRISTIN S. REPYAK, AND PAMELA J. KLING
}

Department of Pediatrics, University of Wisconsin-Madison School of Medicine and Public Health, Madison, Wisconsin 53792; Meriter Hospital, Madison, Wisconsin 53715

\begin{abstract}
Iron is essential for fetal organ development, but the effect of isolated iron deficiency on nephrogenesis is unknown. Human premature infants are at risk for disrupted nephrogenesis because glomerular development is incomplete until 36-wk gestation. We modeled the effects of iron on postnatal glomerulogenesis in four groups of immature rats from P4 to P12: dam fed controls (DF), dam fed with sham gastrostomy surgery (DF + SS), iron-deficiency anemia (IDA), fed iron-deficient formula through gastrostomy apart from the dam, and IDA plus simultaneous enteral iron rescue $(\mathrm{IDA}+\mathrm{Fe})$. Hematocrit, plasma ferritin, and body and kidney tissue iron contents were measured. Tissue was examined. Rats grew similarly, but IDA rats exhibited lower hematocrit, plasma ferritin, and body and kidney iron contents than DF, DF + SS, or IDA + Fe. IDA exhibited 1.7 fewer radial glomerular counts (RGCs), 26\% reduced glomerular density, and $29 \%$ less planar glomerular surface area than DF, with partial improvement in IDA + Fe. Compared with $\mathrm{DF}$ or DF $+\mathrm{SS}$, we observed elevated plasma CRP levels and tubulointerstitial fibrosis in the IDA and IDA $+\mathrm{Fe}$ groups. IDA reduced glomerular density, glomerular surface area, and promoted fibrosis. Iron substantially rescued renal growth and development, supporting the critical role of iron in late nephrogenesis. (Pediatr Res 66: 619-624, 2009)
\end{abstract}

$I^{n}$ ron is essential to human fetal metabolism, growth, and development (1). Although the clinical effects of iron deficiency are best studied in the brain, iron is needed for energy, cell proliferation, mitochondrial function, nerve development, and neurotransmitter function in all tissues $(2,3)$. Fetal and newborn periods are characterized by increased susceptibility to tissue iron depletion because of high iron needs for rapid cellular growth (4). In the last trimester of term human pregnancies, the fetus acquires two thirds of its birth weight, body iron stores (4), and kidney nephrons (5). Because conditions associated with human fetal growth restriction are also associated with decreased tissue iron content (6) and decreased nephron number (7), we sought a model to examine iron deficiency and renal development with normal somatic growth.

Before 36 wks gestation, in or ex utero nutritional factors (protein restriction and maternal vitamin A deficiency), metabolic factors (gestational diabetes, intrauterine growth retardation, and glucocorticoid exposure) and toxic factors (neph-

Received February 9, 2009; accepted August 25, 2009.

Correspondence: Pamela J. Kling, MD, Department of Pediatrics, University of Wisconsin and Meriter Hospital, 202 S Park Street, 6 Center, Madison, WI 53715; e-mail: pkling@pediatrics.wisc.edu

Supported by Thrasher Research Fund (P.J.K.), UW Medical Student Shapiro Research Fund (K.A.D.), UW Medical Student Cardiovascular Research Center Grant (K.A.D.), and UW Undergraduate Hilldale Research Fellowship (M.J.S.) for funding. rotoxic drugs) are linked to lower nephron numbers, reviewed in Zandi-Nejad et al. (8). Animal studies confirm that nutritional iron deficiency during gestation results in hypertensive offspring (9-12); but these models are sufficiently extreme to restrict normal intrauterine growth. Because of global growth and metabolic disturbances, fetal glucocorticoid pathways are disturbed in antenatal models of gestational iron deficiency, but antenatal glucocorticoid administration alone decreases birth weight and nephron numbers (8). Examining nephron number is important because it is linked to the development of essential hypertension as an adult (9-12). Beginning postnatal life with reduced nephron allotment can be associated with increased sodium retention, glomerular hyperfiltration, and eventually deterioration into hypertension (13). However, much remains to be known about the mechanism of altered fetal environment and reduced nephron allotment. We hypothesize that iron plays a critical and an independent role in normal renal development. Because newborn rats are similar to premature humans and undergo postnatal nephrogenesis, our goal was to examine nephrogenesis in a novel rat model of iron deficiency with normal somatic growth, to determine whether iron deficiency is mechanistically linked to deficient nephron numbers.

\section{METHODS}

Animals. The UW-Madison Animal Care and Use Committee approved this protocol. Female timed-pregnant Sprague-Dawley rats (Charles River Laboratories, Wilmington, MA) were fed iron-sufficient basic rodent diet (Harlan-Teklad 8604, $325 \mathrm{mg} / \mathrm{kg}$ iron) and delivered at term. At least three dams were bred to deliver on the same day. At postnatal d 1 (P1), these litters were culled to 10 to standardize growth and development. Ten pups from this pool of 30 were randomly returned to each of the dams to mix up the litters. At P4, groups were again randomly assigned and studied: (1) iron-sufficient dam fed controls raised with the dam (DF), (2) DF experiencing sham gastrostomy surgery at P4 (DF + SS) as a dam-reared surgical control, (3) iron-deficiency anemia, raised away from the dam, fed a low-iron artificial formula through gastrostomy starting at P4 (IDA), and (4) IDA animals gastrostomy fed, but simultaneously treated with daily oral iron supplements $($ IDA $+\mathrm{Fe}$ ) with the intent to rescue from IDA. DF + SS, IDA, and IDA + $\mathrm{Fe}$ groups underwent gastrostomy at P4. After anesthesia with isoflurane, a 16-gauge IV catheter was inserted into the greater curvature of the stomach. The catheter was immediately removed in DF + SS group. In IDA and IDA + Fe groups, a polyethylene PE-10 tubing (Clay Adams, Parsippany, NJ) with one end heat flared was inserted through the catheter sleeve into the stomach, and IV catheter removed (14). The cannulas were fitted to PE-50 tubing attached to syringes mounted on variable speed (Genie-8, Kent Scientific, Torrington, CT) infusion pumps, housed in a bench-top refrigerator at $4^{\circ} \mathrm{C}$ and programmed to infuse $20 \mathrm{~min} / \mathrm{h}$ (14). IDA and IDA + Fe rats were housed in

\footnotetext{
Abbreviations: DF, dam fed; DF + SS, dam fed + sham surgery; IDA, iron-deficiency anemia; IDA + Fe, iron deficiency + iron; P, postnatal; RGC, radial glomerular count; ZnPP/H, zinc protoporphyrin/heme
} 
individual, pint-sized, vented polypropylene cups floating in a $40^{\circ} \mathrm{C}$ water bath (Precision, Chicago, IL). After recuperation, Pedialyte (Abbott Laboratories, Sturgis, MI) and then low iron rat milk substitute (RMS) were infused through the cannula. The infusion rate of RMS was adjusted daily to match the normal weight gain of a suckling litter size of $10\left(370 \mu \mathrm{L} \cdot \mathrm{g}^{-1} \cdot \mathrm{d}^{-1}\right)$ based on mean daily group weight (14). IDA and IDA + Fe rats were handled at least three times daily for manual urination and defecation. In IDA and IDA $+\mathrm{Fe}$, RMS, a low iron formula $\left(4.8 \mathrm{mg} \cdot \mathrm{L}^{-1}\right.$ elemental iron component), was administered from P4 to P12. Rats were either treated with oral ferrous sulfate Fer-In-Sol Drops (Mead Johnson, Evansville, IN) at $6 \mathrm{mg} \cdot \mathrm{kg}^{-1} \cdot \mathrm{d}^{-1}$ elemental iron (IDA $+\mathrm{Fe}$ ) or equal sham volume of PBS (DF, DF + SS, IDA). Fer-In-Sol dose was based on preliminary data showing normalization of $\mathrm{Hb}$ $(15,16)$. After surgery, DF + SS pups were kept warm until recovered and active then returned to the dam. All groups were handled at least three times daily to match gastrostomy groups. In all groups, weight and tail length, as an index of skeletal growth, were recorded. On P12, all pups were killed by lethal isoflurane for blood/tissue harvest.

IDA diet. The evaporated cow's milk protein-based formula also has $20 \%$ intralipid (Baxter Scientific, Deerfield, IL), added multivitamins, minerals, salts, and sunflower oil. RMS contained $24 \mathrm{mg} \cdot \mathrm{L}^{-1}$ ferrous sulfate/7H20, or $4.8 \mathrm{mg} \cdot \mathrm{L}^{-1}$ elemental iron component (14), an amount $40 \%$ of that contained in current US human formulas.

Confirming the model of IDA. Blood samples were collected in EDTA microtubes after cardiac puncture. To assess for anemia, complete blood counts were measured by PocH100i Analyzer (Sysmex, Mundelien, IL) and reticulocytes manually counted. Whole blood was centrifuged at $3000 \mathrm{rpm}$ for 5 min, plasma removed, and plasma frozen at $-80^{\circ} \mathrm{C}$ for later assays, including plasma ferritin (Rat Ferritin ELISA, Immunology Consultants, Newberg, OR) and plasma C-reactive protein (CRP) ELISA, Life Diagnostics, West Chester, PA. Plasma ferritin levels were obtained as a proxy of storage iron. Plasma CRP levels were included to aid interpretation of plasma ferritin, as both rise as acute phase reactants. $\mathrm{ZnPP} / \mathrm{H}$ was measured on the cell pellet after rinsing to remove interfering pigments (17) with a 206D hematofluorometer (Aviv Biomedical, Lakewood, NJ). In humans and rats, $\mathrm{ZnPP} / \mathrm{H}$ ratio measures incomplete iron incorporation into $\mathrm{Hb}$, with zinc substituting for iron with poor iron availability, and less affected by inflammation than plasma ferritin (16-18).

Tissue weight and iron content methods. Bilateral wet kidney weight was determined at sacrifice. In a subset of animals, left kidneys were collected for iron analysis. In a subset of animals designated for body iron analysis, no blood draws were performed. Body iron content was determined after removing gastrointestinal tracts (esophagus to colon) to eliminate any unabsorbed, intraluminal iron from any remaining oral iron supplements. Carcasses or kidneys were weighed and iron content determined by acid digestion and atomic absorption (19). Tissue samples were placed in Teflon tubes engineered at UW Department of Engineering for acid digestion. Nitric then perchloric acids were added and tubes brought up to the same volume with $3 \mathrm{M} \mathrm{HCl}$ (19). Iron standards and samples were read using an atomic absorption flame spectrophotometer (Perkin Elmer 2280, Waltham, MA).

Kidney histologic methods. Right kidneys were placed in 10\% formalin (Fisher Scientific, Fairlawn, NJ) at sacrifice and paraffin blocks prepared. Ten-micrometer sections were cut and stained with hematoxylin and eosin for anatomy and Gomori trichrome (both Fisher Scientific) and reticulin (Richard-Allan Scientific, Kalamazoo, MI, counterstained with nuclear red (Fisher Scientific) for collagen analysis. Photomicrographs were digitally captured by a SPOT Insight Camera (Diagnostic Instruments, Sterling Heights, MI) and a Nikon Eclipse 50i/55i (Melville, NY).

Morphometric parameters were blindly determined using SPOT Advanced software, Diagnostic Instruments, Sterling Heights, MI. Radial glomerular counts (RGCs) were determined on mid-longitudinal sections as mean glomerular generations from eight different $10 \times$ photomicrograph fields of kidney cortex, with care to include the corticomedullary junction $(20,21)$. Generations were identified by drawing parallel lines perpendicular to a mid-kidney medullary ray starting at the first glomerulus seen at the corticomedullary junction, continuing the process to the capsule, and ensuring every glomerulus traverses a line (20). In mid-longitudinal sections, glomerular density (average number of glomeruli per $\mathrm{mm}^{2}$ of cortex) was determined in 10 fields at $10 \times$. Mean glomerular planar surface area (average glomerular area in $\mu \mathrm{m}^{2}$ per cortex area in $\mu \mathrm{m}^{2}$ ) was determined by outlining all identifiable glomeruli, excluding the Bowman's space, from 10 fields at $10 \times$.

On Gomori trichrome-stained longitudinal sections, exhaustive photomicrographs of the corticomedullary junction at $20 \times$ were analyzed for type I collagen fibers staining teal color with phosphotungstic acid. Metamorph 6.1 software (Molecular Devices, Downingtown, PA) quantified the teal blue color of stained collagen fibers reported as an average percent fibrosis per photomicrograph. On reticulum-stained longitudinal sections, $20 \times$ photomicrographs were obtained to qualitatively display the black color of type III collagen.

Statistical analysis. Findings from DF, DF + SS, IDA, and IDA + Fe groups were compared by Factorial ANOVA, with Fisher post hoc test for parametric and Kruskall-Wallis for nonparametric data. Sex differences were examined by unpaired $t$ testing or Mann-Whitney $U$ testing. $p<0.05$ was considered significant.

\section{RESULTS}

Confirmation of IDA model. P12 rats fed iron-deficient formula for $8 \mathrm{~d}$ were anemic and exhibited hematologic parameters consistent with iron deficiency. Weights were the same in all groups (Table 1). Although tail length (linear growth) was slightly shorter in the DF + SS and IDA groups, the ratio of weight/tail length was similar in all groups. For hematologic and anthropometric parameters, between 10 and 20 values in each group were obtained. Because of double randomization procedure at $\mathrm{P} 1$ and $\mathrm{P} 4$, pups from 6 to 9 different pregnancies were used for hematologic and anthropometric parameters. In addition, we analyzed tissue iron studies and histologic parameters from 5 to 9 samples per group, using pups from 3 to 6 different pregnancies. $\mathrm{Hb}$ and hematocrit represent erythropoiesis and are shown in Table 1. $\mathrm{Hb}$ was lower in IDA and DF + SS than in DF or IDA + Fe, but hematocrits were lower only in IDA, compared with the other groups. Consistent with iron deficiency, erythrocyte

Table 1. Anthropometric and hematological parameters at P12

\begin{tabular}{|c|c|c|c|c|}
\hline & DF & $\mathrm{DF}+\mathrm{SS}$ & IDA & $\mathrm{IDA}+\mathrm{Fe}$ \\
\hline Weight $(\mathrm{g})$ & $27.7(3.4)$ & $24.0(2.9)$ & $26.0(4.0)$ & $25.4(3.7)$ \\
\hline Tail length (mm) & $44.9(2.9)$ & $42.4(2.8)^{*}$ & $42.3(2.0)^{*}$ & $43.1(2.1)$ \\
\hline Weight/tail length & $0.613(0.066)$ & $0.567(0.068)$ & $0.585(0.073)$ & $0.593(0.064)$ \\
\hline Relative wet kidney (wt mg/g) & $14.4(4.3)$ & $14.3(2.2)$ & $15.2(1.6)$ & $14.9(1.9)$ \\
\hline Hemoglobin $(g / L)$ & $95.3(8.4)$ & $83.9(11.1)^{*}$ & $81.9(5.2)^{*}$ & $96.3(14.9) \dagger+$ \\
\hline Absolute reticulocyte $\left(10^{12} / \mathrm{L}\right)$ & $1.09(0.20)$ & $1.09(0.23)$ & $1.18(0.30)$ & $21.51(0.31)^{* \dagger+}$ \\
\hline Hematocrit & $29.0(2.3)$ & $27.6(3.7)$ & $25.7(3.3)^{*}$ & $30.1(1.1) \ddagger$ \\
\hline $\operatorname{MCV}(f L)$ & $83.6(4.9)$ & $86.3(3.2)$ & $78.9(2.4)^{* \dagger}$ & $84.3(3.2) \div$ \\
\hline RDW (\%) & $16.4(2)$. & $16.7(1.4)$ & $21.4(2.2)^{* \dagger}$ & $18.5(1.9)^{* \dagger \dagger}$ \\
\hline $\mathrm{ZnPP} / \mathrm{H}(\mu \mathrm{mol} / \mathrm{mol})$ & $77.8(26.7)$ & $93.2(24.2)$ & $135.9(30.8)^{* \dagger}$ & $108.1(22.1)^{*} \ddagger$ \\
\hline
\end{tabular}

Mean (SD).

* Differs from DF.

$\dagger$ Differs from DF + SS.

\$Differs from IDA. 
indices were abnormal only in the IDA group. $\mathrm{ZnPP} / \mathrm{H}$ in IDA + Fe was partially corrected (Table 1). In the four treatment groups, no sex differences were seen in the erythropoietic parameters. In Figure $1 A-C$, body iron, plasma ferritin levels, and kidney iron confirm tissue iron deficiency in only IDA, with rescue occurring in IDA $+\mathrm{Fe}$. Body iron, kidney iron, and plasma ferritin in DF + SS approximated DF control levels. The inflammatory index, plasma CRP (Fig. 1D), was higher in IDA and IDA + Fe than DF or DF + SS.

Kidney histology analyses. Mid-longitudinal sections are reported here, as preliminary work showed this orientation had optimal visualization for RGC determinations and the measurements for glomerular density, mean glomerular surface area, and glomerular planar surface area were similar to systematic, more exhaustive cross-sectional measurements. Because renal glomerular generations radiate from the center of the kidney with increasing maturation, RGCs were first determined on control rats at different stages of development (Fig. 2). Glomerular generations increased in number from approximately 6 at birth to 10 at sacrifice. On $2.5 \times$ views (2A), darker capsule in P8 suggesting a small residual nephrogenic zone no longer present on P12. Lines on $10 \times$ photomicrographs show greater number of RGCs in P20, compared with other time points (2B). Because glomeruli develop in successive generations $(20,21)$ from epithelial branching until P10, we examined and found 1.7 fewer RGCs from IDA, compared with DF, DF + SS, or IDA + Fe rats at P12 (Fig. $3)$. Figure $4 A$ shows an example of computer-generated glomerular measurements on photomicrographs. Figure $4 B$ shows $26 \%$ lower mean glomerular density and Figure $4 C$ shows a $29 \%$ lower mean glomerular planar area of ID, compared with $\mathrm{DF}$ or DF + SS. ID + Fe exhibited similar glomerular density to control, but glomerular planar surface area was similar to IDA. In histologic analyses, numbers were insufficient to examine for sex differences.

Interstitial fibrosis analysis. Figure 5 shows Gomori trichrome teal blue (collagen I) and reticulin black (collagen III) staining of all groups. Arrows on $A$ and $B$ (lower power)
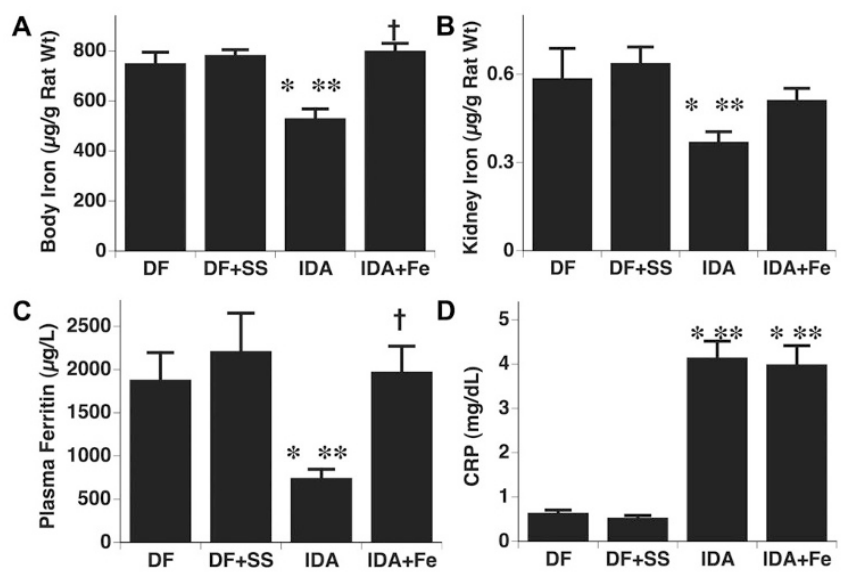

Figure 1. Tissue iron depletion was observed. IDA rats at P12 exhibited $(A)$ lower body iron content $(\mu \mathrm{g} / \mathrm{g}$ of rat $\mathrm{wt}),(B)$ lower kidney iron content corrected for rat wt $(\mu \mathrm{g} / \mathrm{g}$ rat wt), $(C)$ lower plasma ferritin levels $(\mu \mathrm{g} / \mathrm{L})$, and $(D)$ higher C-reactive protein (CRP in $\mathrm{mg} / \mathrm{dL})$. *Differ from DF; **differ from $\mathrm{DF}+\mathrm{SS}$; †rom IDA.

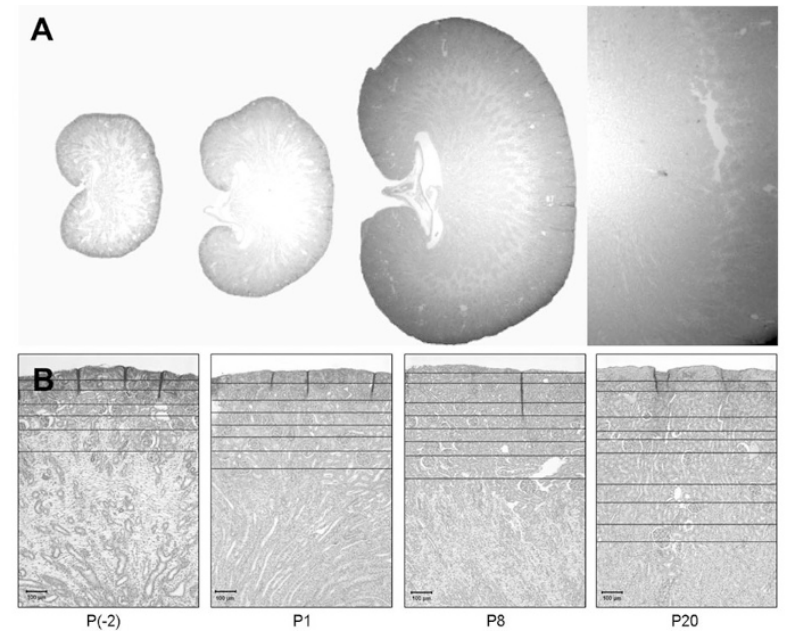

Figure 2. In renal branching morphogenesis, glomeruli develop in successive generations that arcade out toward the renal capsule. Kidneys in longitudinal sections at $\times 2.5(A)$, showing perinatal nephrogenesis $\mathrm{P}(-2), \mathrm{P} 1, \mathrm{P} 8$, and P20 in untreated rats, with $(B)$ showing the corresponding views with linear marking of radial glomerular counts (RGCs) as kidneys mature. Bars at the bottom indicate $100 \mu \mathrm{m}$.

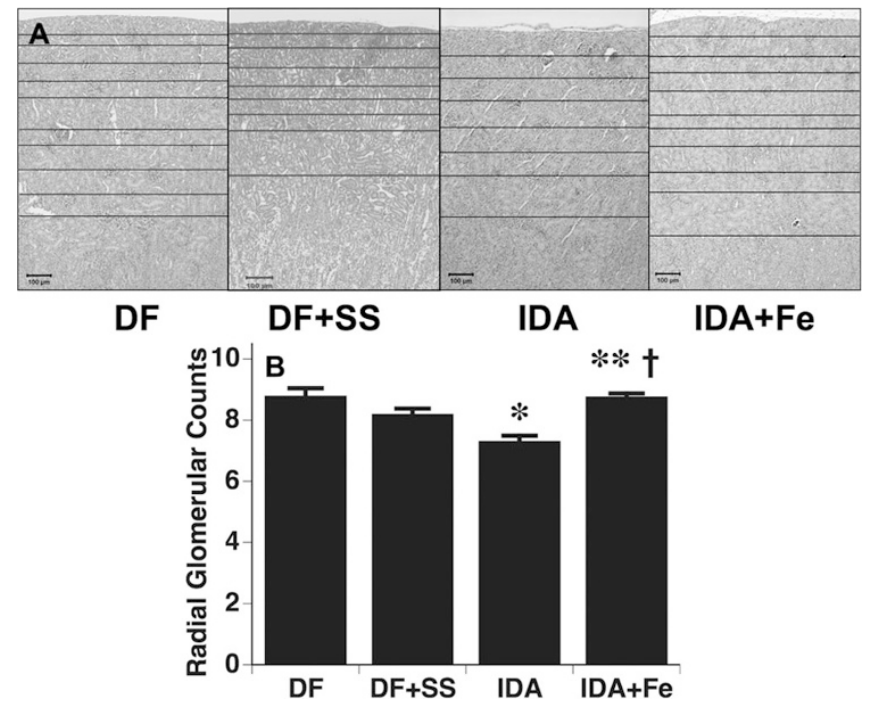

Figure 3. Iron deficiency decreased RGCs at P12. RGCs $(A)$ in DF, DF + SS, IDA, and IDA + Fe are shown. Bars indicate $100 \mu \mathrm{m}$. Graphically $(B)$, lower RGCs are seen in IDA, compared with DF, DF + SS and IDA + Fe. $*$ Differ from DF; **differ from DF $+\mathrm{SS}$; †from IDA.

indicate increased corticomedullary collagen staining for IDA and IDA + Fe, compared with DF or DF + SS. Oil immersion views $(C$ and $D)$ support increased collagen around the corticomedullary glomeruli in the IDA and IDA + Fe groups. On exhaustive digital photomicrographs of Gomori trichromestained slides, collagen was morphometrically quantified, compared with background surface area, showing 30-40\% greater staining for fibrosis seen in IDA and IDA $+\mathrm{Fe}$ $(0.96-1.03 \%)$ than DF or DF + SS $(0.57-0.65 \%), p<0.001$.

\section{DISCUSSION}

This is the first study showing abnormal renal development in newborn rats after normal gestation and postnatal growth, but isolated postnatal dietary iron deficiency. Renal develop- 


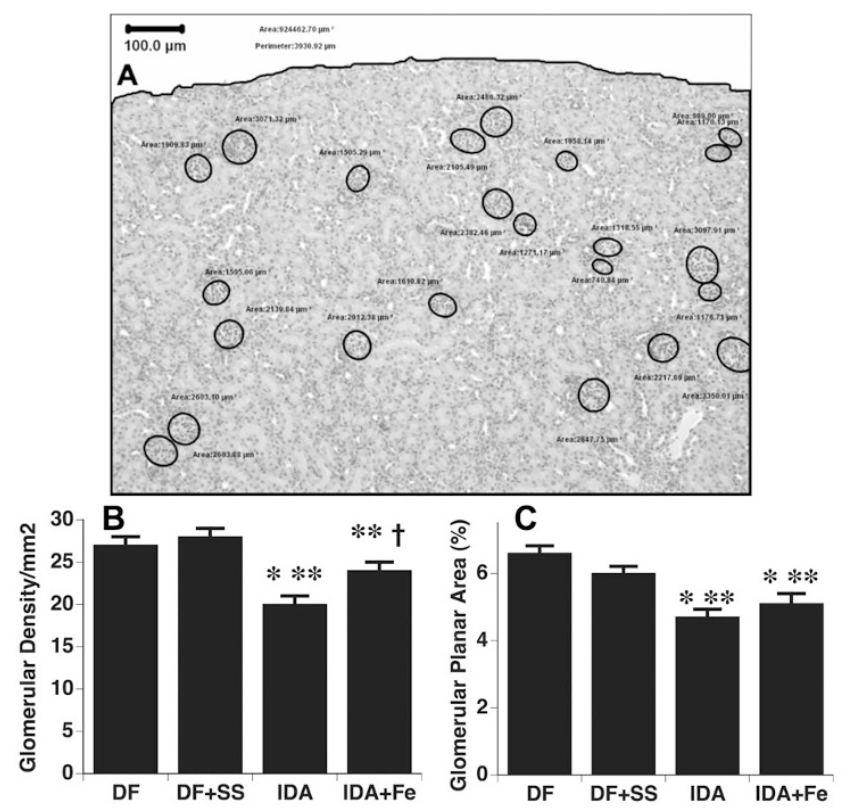

Figure 4. Example of $(A)$ digital outlining of glomeruli, calculation of photomicrograph area, calculation of glomerular, and total surface area in a DF rat at P12. Slides were oriented with renal capsule at the top, as shown in the figure. These calculations account for glomerular density $/ \mathrm{mm}^{2}$, glomerular planar surface area (\% cortex). At P12, glomerular density was lower in IDA, compared with DF, DF + SS and IDA + Fe $(B)$ and glomerular planar surface area $(C)$ was lower in IDA, compared with DF, DF + SS. *Differ from DF; **differ from DF + SS; †from IDA.
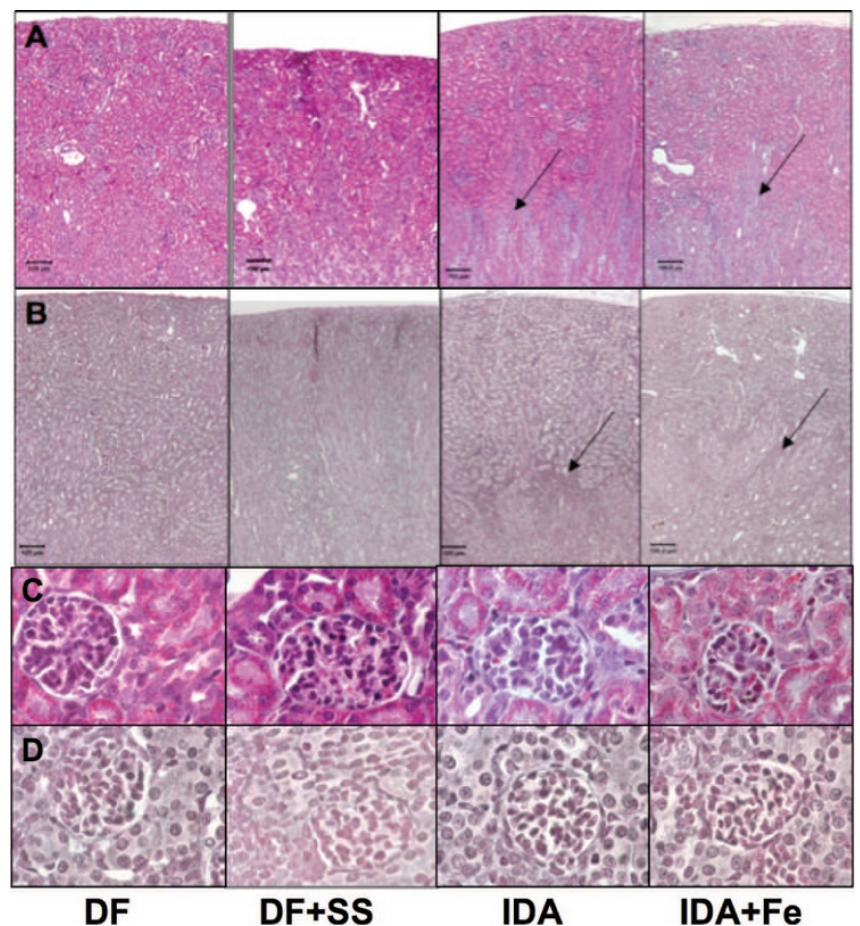

Figure 5. Staining for collagen. At P12, the corticomedullary junction $A$ and $C$ show Gomori trichrome staining for collagen $\mathrm{I}$ and $B$ and $D$ reticulin staining for collagen III. Bars indicate $100 \mu \mathrm{m}$. Compared with DF and DF + SS $(A)$, there was quantitatively increased expression of collagen I in IDA and IDA $+\mathrm{Fe}, p<0.001$, but no difference between IDA and IDA $+\mathrm{Fe}$. Qualitatively, increased reticulin staining is also seen in IDA and IDA + Fe. Bars indicate $100 \mu \mathrm{m}$. Higher power views of Gomori trichrome $(C)$ and reticulin $(D)$, for each group, oil immersion. ment in humans continues though 36-wk gestation, with approximately two thirds of nephrons developing during the last trimester $(5,7)$. In fetuses or premature humans, disturbances in nephrogenesis permanently decrease nephron allotment $(20,21)$. Comparable with premature humans, we confirmed that renal development in rats was immature at birth, with generations developing from the nephrogenic zone ex utero, allowing us to independently model the pathophysiology of tissue iron deficiency during a developmental time point equivalent to late gestation or premature human life.

The IDA model exhibited anemia and iron deficiency, despite normal body weight and linear growth. Although mean tail length, representing linear growth, was slightly shorter in the DF + SS and IDA groups, the ratio of body weight/tail length was similar in all groups. Our findings are consistent with the literature, describing preferential diversion of iron to erythropoiesis $(22,23)$. Compared with DF, our nutritional irondeficiency model exhibited a slightly lower (13\%) Hb iron, despite a $46 \%$ lower renal iron and $30 \%$ lower total body iron. A second control group included to account for surgical stresses exhibited a slightly lower $\mathrm{Hb}$, but normal hematocrit, erythrocyte indices, $\mathrm{ZnPP} / \mathrm{H}$, plasma ferritin, and body and kidney iron contents, supporting normal iron in that group. Our data support the assumption that in early development, when iron supply is limited, iron was preferentially directed to erythrocytes at the expense of the kidneys.

We found newborn rats made nutritionally iron deficient during late nephrogenesis demonstrated similar kidney weight, but abnormal renal development, with fewer glomerular generations, reduced cortical nephron density, and decreased total glomerular planar surface area. Models of gestational iron deficiency are linked to hypertension as offspring mature, but fetal growth restriction is a universal finding in these models $(9-11)$. Although global nutrient restriction during rat gestation clearly disrupts fetal renal histologic architecture $(24,25)$, only one published study examined renal histology in offspring after a model of isolated iron deficiency during gestation (12). The authors found $23 \%$ fewer nephrons, $15 \%$ smaller relative kidney size based on birth weight, and $15 \%$ smaller rats (12), making it difficult to discern the independent effects of iron deficiency versus other global metabolic effects involved in growth restriction.

In postnatal rats, the effects of global nutritional deprivation on renal development were examined in only one published study that doubled suckling litter size (26). Rats were $25 \%$ smaller during late nephrogenesis (P7-10), ultimately exhibiting 25\% fewer total nephrons into adulthood (26). In comparison, we observed a similar decrease in nephron numbers, but normal body size and proportionally normal kidney size.

We observed no sex differences in anemia, body, or renal iron content. Male animals with decreased nephron allotment ultimately develop hypertension at an earlier postnatal age, based on the assumption that sex hormones impact development of hypertension (25). However, at least one fetal nutrient restriction study found male lambs exhibit fewer nephrons than females at birth (27). Our numbers were insufficient to examine for sex differences in histologic renal development, but male and 
female animals were balanced. It would be important to examine sex differences in our postnatal model (28).

In normal dam-reared rats at P4, we previously observed a physiologic nadir in relative body iron content $(15,19)$, due to no appreciable net iron absorption before P4. Because of the tenuous iron status at $\mathrm{P} 4$, we simultaneously rescued iron status by daily gavaged ferrous sulfate and restored body iron, $\mathrm{Hb}, \mathrm{RBC}$, and erythrocyte indices, glomerular generations and nephron density, supporting the independent contribution of iron in late nephrogenesis. Iron was previously shown to be important in early renal ureteric bud formation (29-31), but iron's role in later nephrogenesis was previously unknown.

Iron supplementation restored body stores (by tissue content and plasma ferritin), but an artifactual rise in plasma ferritin may be seen when CRP levels are also higher. Iron treatment only exhibited an intermediate restoration of kidney iron and glomerular planar surface area, supporting a role for other factors, i.e., relatively lower tissue iron bioavailability or irreversible histologic changes. In humans without overt renal disease, elevated CRP predicts the ultimate development of hypertension and decreased renal function (32). Of significance, elevated CRP levels paralleled the microscopic corticomedullary fibrotic changes in IDA and IDA $+\mathrm{Fe}$. Because the corticomedullary junction contains the most mature glomeruli (33), it is possible that body iron was restored, but kidney iron was either insufficient or transport delayed, resulting in an irreversible fibrosis from an epithelial-tomesenchymal transdifferentiation (34-36). Fibrosis can occur in response to tissue hypoxia through hypoxia-inducible factor mechanisms known to up-regulate profibrotic inflammatory factors (34-36). The corticomedullary junction is most vulnerable to hypoxia due to greater metabolic activity and lower oxygen tension (36). Studies cannot definitively distinguish whether histologic changes are from iron deficiency or hypoxia, because on a cellular basis, iron deficiency also mimics hypoxia $(37,38)$, activating the hypoxia-inducible factor and up-regulating downstream profibrotic genes. Although severe anemia alone may cause tissue hypoxia, anemia was mild in IDA rats. Previous work shows abnormal renal function is extremely uncommon in severe isoimmune hemolytic anemia in the human fetus (39), an important observation supporting the linkage of iron-specific pathways to abnormal nephrogenesis in our model. Although we obtained CRP levels to guide interpretation of plasma ferritin, we found these values strikingly linked the treatments showing corticomedullary fibrosis. Because CRP correlated to interstitial fibrosis and previous work showed that elevated CRP levels in humans predict hypertension and renal insufficiency (32), this may explain why hypertension is not always seen with lower nephron allotment, but results from the combination of oligonephropathy and fibrotic injury (25).

Using a surgical control showed that surgery did not contribute to kidney fibrosis because this group is similar to DF for most experimental findings, including lower CRP and absence of fibrosis. This leaves the diet (either something missing or present in the diet) most likely responsible for the architectural corticomedullary disturbance. The DF + SS group's lower $\mathrm{Hb}$ was unexpected but may be due to blood loss from the procedure because it was not associated with inflammation or iron deficiency. It also supports that mildly decreased oxygen availability in the absence of iron deficiency barely impacts kidney development.

Limitations of the study include inability to perform exhaustive nephron counting or the dissector method $(24,40)$, but examining the full length of the kidney in a mid-sagittal plane and with such as striking difference between groups, findings would be unlikely to be different. Another limitation is our sampling at only one point in time (P12), and more points in time can be studied. Only one iron dosage, 6 $\mathrm{mg} \cdot \mathrm{kg}^{-1} \cdot \mathrm{d}^{-1}$ of ferrous sulfate, was used because previously that dose restored $\mathrm{Hb}(15,19)$. Another dosage or form of iron may exhibit better bioavailability and organ trafficking. Although gastrostomy does not seem to cause intestinal water retention or inflammation, artificial formula has been shown to cause hyperplasia of intestinal villi and accelerated maturation of enzyme activity (14). Cow's milk proteins or iron supplementation may contribute to elevated CRP because it alters intestinal flora $(41,42)$.

Our study differs from the published fetal studies limiting maternal iron intake during pregnancy because these are associated with poor growth and other major metabolic disturbances $(9-11)$. This is the first study showing abnormal renal development accompanying normal gestational environment and somatic growth in iron-deficient animals. Our gastrostomy model removes some major metabolic and adrenocorticoid disturbances accompanying fetal growth restriction, a distinction important because adrenocorticoid disturbances are linked to impaired nephrogenesis and developmental programming of adult hypertension (43). The sham surgery group showed that surgery is a minor contributor to impaired nephrogenesis, with no effect on nephron density or glomerular planar surface area. Although we cannot speculate beyond P12 as to whether hypertension develops, our model is well poised for future experimentation allowing pups to mature, to determine whether the decreased nephron allotment and/or fibrosis lead to abnormal renal angiotensin physiology, abnormal sodium handling, hyperfiltration, and then hypertension. This model of developmental programming has important clinical implications for premature infants born with poor iron stores who are similarly exposed to indwelling catheters, tube insertions, and insufficient iron supplementation during glomerulogenesis.

Acknowledgments. We thank the technical support of Don Singer, MD, Michael Georgieff, MD, Suann S. Woodward, Kelsey J. Kleven, Melinda E. Chen, Debra Schneider, and Satoshi Kinoshita.

\section{REFERENCES}

1. Allen LH 2000 Anemia and iron deficiency: effects on pregnancy outcome. Am J Clin Nutr 71:1280S-1284S

2. Georgieff MK 2007 Nutrition and the developing brain: nutrient priorities and measurement. Am J Clin Nutr 85:614S-620S

3. Georgieff MK 2006 Iron. In: Hay WH Jr, Thureen P (eds). Neonatal Nutrition and Metabolism. Cambridge University Press, Cambridge, UK, pp 291-298

4. Lozoff B, Kaciroti N, Walter T 2006 Iron deficiency in infancy: applying a physiologic framework for prediction. Am J Clin Nutr 84:1412-1421 
5. Chevalier RL 1996 Developmental renal physiology of the low birth weight pre-term newborn. J Urol 156:714-719

6. Chockalingam UM, Murphy E, Ophoven JC, Weisdorf SA, Georgieff MK 1987 Cord transferrin and ferritin values in newborn infants at risk for prenatal uteroplacental insufficiency and chronic hypoxia. J Pediatr 111:283-286

7. Hinchliffe SA, Lynch MR, Sargent PH, Howard CV, Van Velzen D 1992 The effect of intrauterine growth retardation on the development of renal nephrons. Br J Obstet Gynaecol 99:296-301

8. Zandi-Nejad K, Luyckx VA, Brenner BM 2006 Adult hypertension and kidney disease: the role of fetal programming. Hypertension 47:502-508

9. Gambling L, Dunford S, Wallace DI, Zuur G, Solanky N, Srai SK, McArdle HJ 2003 Iron deficiency during pregnancy affects postnatal blood pressure in the rat. J Physiol 552:603-610

10. Lewis RM, Petry CJ, Ozanne SE, Hales CN 2001 Effects of maternal iron restriction in the rat on blood pressure, glucose tolerance, and serum lipids in the 3-month-old offspring. Metabolism 50:562-567

11. Crowe C, Dandekar P, Fox M, Dhingra K, Bennet L, Hanson MA 1995 The effects of anaemia on heart, placenta and body weight, and blood pressure in fetal and neonatal rats. J Physiol 488:515-519

12. Lisle SJ, Lewis RM, Petry CJ, Ozanne SE, Hales CN, Forhead AJ 2003 Effect of maternal iron restriction during pregnancy on renal morphology in the adult rat offspring. Br J Nutr 90:33-39

13. Brenner BM, Lawler EV, Mackenzie HS 1996 The hyperfiltration theory: a paradigm shift in nephrology. Kidney Int 49:1774-1777

14. Dvorak B, McWilliam DL, Williams CS, Dominguez JA, Machen NW, McCuskey RS, Philipps AF 2000 Artificial formula induces precocious maturation of the smal intestine of artificially reared suckling rats. J Pediatr Gastroenterol Nutr 31:162-169

15. Dubuque SH, Dvorak B, Woodward SS, McCuskey RS, Kling PJ 2002 Iron-deficient erythropoiesis in neonatal rats. Biol Neonate 81:51-57

16. Kling PJ, Willeitner A, Dvorak B, Blohowiak SE 2008 Enteral erythropoietin and iron stimulate erythropoiesis in suckling rats. J Pediatr Gastroenterol Nutr 46:202207

17. Hastka J, Lasserre JJ, Schwarzbeck A, Strauch M, Hehlmann R 1992 Washing erythrocytes to remove interferents in measurements of zinc protoporphyrin by front-face hematofluorometry. Clin Chem 38:2184-2189

18. Labbe RF, Vreman HJ, Stevenson DK 1999 Zinc protoporphyrin: a metabolite with a mission. Clin Chem 45:2060-2072

19. Blohowiak SE, Chen ME, Repyak KS, Baumann-Blackmore NL, Carlton DP, Georgeiff MK, Crenshaw TD, Kling PJ 2008 Reticulocyte enrichment of zinc protoporphyrin/heme ratios in human cord blood and suckling rats. Pediatr Res 64:63-67

20. Rodriguez MM, Gomez AH, Abitol CL, Chandar JJ, Duara S, Zilleruelo GE 2004 Histomorphometric analysis of postnatal glomerulogenesis in extremely preterm infants. Pediatr Dev Pathol 7:17-25

21. Rodriguez MM, Gomez A, Abitbol C, Chandar J, Montane B, Zilleruelo G 2005 Comparative renal histomorphometry: a case study of oligonephropathy of prematurity. Pediatr Nephrol 20:945-949

22. Georgieff MK, Schmidt RL, Mills MM, Radmer WJ, Widness JA 1992 Fetal iron and cytochrome c status after intrauterine hypoxemia and erythropoietin administration. Am J Physiol 262:R485-R491
23. Guiang SF, Georgieff MK, Lambert DJ, Schmidt RL, Widness JA 1997 Intravenous iron supplementation effect on tissue iron and hemoproteins in chronically phlebotomized lambs. Am J Physiol 273:R2124-R2131

24. Schreuder MF, Nyengaard JR, Fodor M, van Wijk JA, Delemarre-van de Waal HA 2005 Glomerular number and function are influenced by spontaneous and induced low birth weight in rats. J Am Soc Nephrol 16:2913-2919

25. Ojeda NB, Grigore D, Alexander BT 2008 Developmental programming of hypertension: insight from animal models of nutritional manipulation. Hypertension 52:44-50

26. Schreuder MF, Nyengaard JR, Remmers F, van Wijk JA, Delemarre-van de Waa HA 2006 Postnatal food restriction in the rat as a model for a low nephron endowment. Am J Physiol Renal Physiol 291:F1104-F1107

27. Gilbert JS, Ford SP, Lang AL, Pahl LR, Drumhiller MC, Babcock SA, Nathanielsz PW, Nijland MJ 2007 Nutrient restriction impairs nephrogenesis in a gender-specific manner in the ovine fetus. Pediatr Res 61:42-47

28. Grigore D, Ojeda NB, Alexander BT 2008 Sex differences in the fetal programming of hypertension. Gend Med 5:S121-S132

29. Landschulz W, Ekblom P 1985 Iron delivery during proliferation and differentiation of kidney tubules. J Biol Chem 260:15580-15584

30. Yang J, Goetz D, Li J-Y, Wang W, Mori K, Setlik D, Du T, Erdjument-Bromage H, Tempst P, Strong R, Barasch J 2002 An iron delivery pathway mediated by a lipocalin. Mol Cell 10:1045-1056

31. Thesleff I, Partanen M-M, Landschulz W, Trowbridge IS, Ekblom P 1985 The role of transferrin receptors and iron delivery in mouse embryonic morphogenesis. Differentiation 30:152-158

32. Vidt DG 2006 Inflammation in renal disease. Am J Cardiol 97:20A-27A

33. Nagata M, Schärer K, Kriz W 1992 Glomerular damage after uninephrectomy in young rats. I. Hypertrophy and distortion of capillary architecture. Kidney Int 42:136-147

34. Norman JT, Clark IM, Garcia PL 2000 Hypoxia promotes fibrogenesis in human renal fibroblasts. Kidney Int 58:2351-2366

35. Maltzman JS, Haase VH 2008 Low oxygen stimulates the immune system. Kidney Int 73:797-799

36. Nangaku M, Eckardt KU 2007 Hypoxia and the HIF system in kidney disease. J Mol Med 85:1325-1330

37. Kling PJ, Dragsten PR, Roberts RA, Dos Santos B, Brooks DJ, Hedlund BE, Taetle $\mathrm{R} 1996$ Iron deprivation increases erythropoietin production in vitro, in normal subjects and patients with malignancy. Br J Haematol 95:241-248

38. Wang GL, Semenza GL 1993 Desferrioxamine induces erythropoietin gene expression and hypoxia-inducible factor-1 DNA-binding activity-implications for models of hypoxia signal transduction. Blood 82:3610-3615

39. Merlob P, Litwin A, Lazar L, Zaizov R 1990 Neonatal ABO incompatibility. Complicated by hemoglobinuria and acute renal failure. Clin Pediatr (Phila) 29:219222

40. Bertram JF 2001 Counting in the kidney. Kidney Int 59:792-796

41. Balmer SE, Wharton BA 1989 Diet and faecal flora in the newborn: breast milk and infant formulas. Arch Dis Child 64:1672-1677

42. Mevissen-Verhage EA, Marcelis JH, Harmsen-Van Amerongen WC, de Vos NM, Verhoef J 1985 Effect of iron on neonatal gut flora during the first three months of life. Eur J Clin Microbiol 4:273-278

43. Vuguin PM 2007 Animal models for small for gestational age and fetal programming of adult disease. Horm Res 68:113-123 